\title{
Research on Image Recognition of Electrical Equipment based on Deconvolution Feature Extraction
}

\author{
Zhe $\mathrm{Li}^{1 *}$, Haifeng $\mathrm{Su}^{2}$ \\ ${ }^{1}$ North China Electric Power University, Department of Electrical Engineering, Lianchi, Baoding, Hebei, China \\ ${ }^{2}$ North China Electric Power University, Department of Electrical Engineering, Lianchi, Baoding, Hebei, China
}

\begin{abstract}
Based on machine learning technology and combining the operation of machine learning from the idea of neural network, this paper focuses on the classification and recognition of image data of transformers, circuit breakers and isolation switches in substations. Firstly, the image enhancement is carried out on the basis of the original image, which simulates the possible scenes in reality. Secondly, using the dual-mode a deconvolutional network to capture significant features from in-depth visible and infrared images. Furthermore, all these features are subjected to the program to conduct transfer learning and weighted fusion. The dual-mode deconvolutional network (DMDN) extracts and highlights the features of the electrical equipment. Compared to traditional model, the recognition accuracy of the improved model is reached at $99.17 \%$.
\end{abstract}

\section{Introduction}

With the rapid development of camera system and Computer vision technology, power system has gradually entered the intelligent monitoring period [1].

At present, many experts and scholars have achieved satisfactory results from conducting in-depth research in the field of image analysis and recognition of electrical equipment, and have achieved satisfactory results. Reference [2] used deep learning model to extract features of power equipment, and then combines with Random Forest classifier to achieve the image recognition of important electrical equipment. Reference [3] constructed a deep convolutional autoencoder network model based on convolutional neural network and combined with autoencoder. The model automatically learns and extracts effective features in small sample images to improve recognition accuracy. Reference [4] proposed an image recognition method for electrical equipment based on the GoogLeNet InceptionV3 model. This method has a high average recognition accuracy for circuit-breakers, current transformers, insulators, arresters and potential transformer. Reference [5] set up the overall objective function for the deconvolution layer and modifies it to a supervised state, and finds the most suitable high-level feature mapping matrix for the training of the back-end convolutional neural network.

In this paper, for small sample data, the data is enhanced, and then the dual-mode deconvolution network (DMDN) is used to extract the features of the visible light and infrared images of the transformer, circuit breaker, and isolating switch, which are pre-set based on the feature extraction of the traditional convolutional neural network. The aim is improving the recognition accuracy using the dual-mode network for overall characteristics of the specific small samples and perform transfer learning and feature fusion on the extracted feature matrix.

\section{Convolutional neural network learning based on dual-mode deconvolution feature extraction}

\subsection{Network structure}

The structure of the convolutional neural network based on dual-mode deconvolution network (DMDN) feature extraction is shown in Figure 1. The network consists of two parts. The first part is the dual-mode deconvolution feature extraction network. Given the visible light and infrared images of the electrical equipment and the randomly initialized feature mapping matrix, the deconvolution network is used for directly performing the decomposition mapping operation from the original image to the hidden layer feature space. At the same time, the decoder reversely maps the hidden layer features back to the input space to reconstruct the input similar to the original image, and differences between the reconstructed image and the original image are used as the optimization target of the objective function. The second part is the recognition training of convolutional neural network. The dual-mode feature mapping matrix learned in the first stage is used as the initial convolution kernel matrix of $\mathrm{CNN}$, and the extracted initial convolution kernel matrix is weighted and fused to realize cross-channel information interaction and integrate, then perform alternate convolution, activation,

\footnotetext{
* Corresponding author: m15561436123@163.com
} 
normalization, and pooling operations (CANP). Finally, the Mini-batch Gradient Descent (MB-SGD) is used to fine-tune $\mathrm{CNN}$ to avoid the vanishing gradient problem during training.
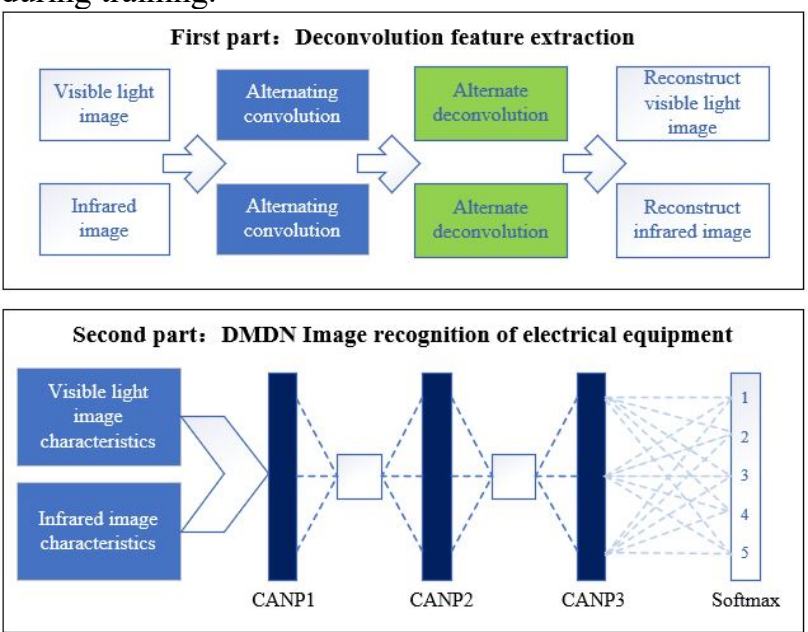

Figure 1. System structure

\subsection{Image feature extraction of electrical equipment}

In this paper, the deconvolution layer is used to stack the hierarchical network model, and the efficient optimization technology is used to extract the feature mapping matrix. The reasoning operation principle of deconvolution network model is shown in Figure 2.

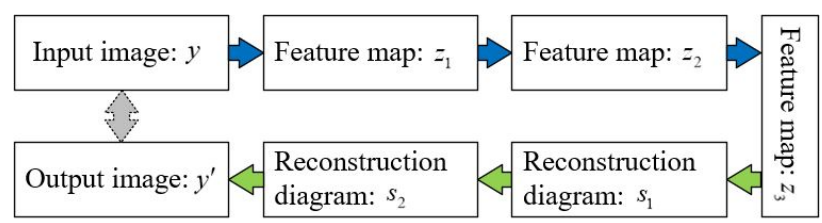

$\Rightarrow$ :convolution $>$ :deconvolution $\quad$ loss

Figure 2. Deconvolution feature extraction

Given an input image and a randomly initialized convolutional neural network, the feature extraction operation is to perform deconvolution on the top feature map $z_{\mathrm{i}}$ to obtain the reconstructed image $s_{\mathrm{i}}$.

Assuming that the image $y$ in the original samples is included in the convolution operation to obtain the feature map, the reconstructed image $s_{\mathrm{i}}$ is obtained by using the deconvolution kernel to do the convolution operation:

$$
S_{i}=\sum_{i=1}^{N_{1}} z_{i}^{l} * f_{i}^{T}
$$

The ultimate goal of feature extraction is to make the reconstructed image and the input image as similar as possible, so the minimum reconstruction error is defined as:

$$
\min C_{1}(\mathrm{y})=\sum_{i=1}^{N}\left\|S_{i}-y_{i}\right\|+\sum_{i=1}^{N_{1}}\left|z_{i}\right|^{1}
$$

The feature learning process of the deconvolution network can be divided into the following two stages: the first stage, given the convolution kernel $f_{\mathrm{i}}$, find the feature map $z_{\mathrm{i}}$ to minimize the objective function. The second stage, given the feature map $z_{\mathrm{i}}$, solve the convolution kernel $f_{\mathrm{i}}$, which minimizes the objective function.

In the specific training process, it is necessary to introduce an auxiliary objective variable $\alpha_{\mathrm{i}}$ for the first time to avoid falling into the local optimum, and then solve the minimum value of the auxiliary objective function $C_{2}(y)$ to make the auxiliary variable and the feature map approximate. The auxiliary objective function is defined as:

$$
C_{2}(y)=\sum_{i=1}^{N}\left\|s_{i}-y_{i}\right\|_{2}^{2}+\sum_{i=1}^{N_{1}}\left\|z_{i}^{l}-\alpha_{i}^{l}\right\|_{2}^{2}+\sum_{i=1}^{N}\left|\alpha_{i}^{l}\right|^{1}
$$

After introducing this auxiliary function, alternately fix the values of $z_{\mathrm{i}}$ and $\alpha_{\mathrm{i}}$ to obtain the optimal solutions respectively. First, fix $z_{\mathrm{i}}$ and solve $\alpha_{\mathrm{i}}$. Derivation of equation (4):

$$
\frac{\partial C_{2}(y)}{\partial z_{i}^{l}}=\sum_{i=1}^{N_{1}}\left(F_{k}^{l}\right)^{T}\left(\sum_{k=1}^{N_{2}} F_{k}^{l} z_{i}^{l}-z_{i}^{l-1}\right)+\mu\left(z_{i}^{l}-\alpha_{i}^{l}\right)
$$

In the stage of finding auxiliary variables $\alpha_{\mathrm{i}}$, if the feature graph $z_{\mathrm{i}}$ is fixed, the optimal problem $\alpha_{\mathrm{i}}$ can be solved by the feature maps. the solution expression $\alpha_{\mathrm{i}}$ of auxiliary variable is:

$$
\alpha_{i}^{l}=\max \left(\left|z_{i}^{l}-\frac{1}{\mu}\right|, 0\right) \frac{z_{i}^{l}}{\left|z_{i}^{l}\right|}
$$

The update process of the feature map $z_{\mathrm{i}}$ is: firstly, given $i$, set equation (4) equal to zero, and solve $z_{\mathrm{i}}$, that is, to solve the following dimensional linear system:

$$
A\left[\begin{array}{c}
z_{1}^{l} \\
\vdots \\
z_{i}^{l}
\end{array}\right]=\left[\begin{array}{c}
\sum_{i=1}^{N_{1}}\left(F_{1, c}^{l}\right)^{T} z_{1}^{l}+\alpha_{1}^{l} \\
\vdots \\
\sum_{i=1}^{N_{1}}\left(F_{i, c}^{l}\right)^{T} z_{i}^{l}+\alpha_{i}^{l}
\end{array}\right]
$$

Where,

$$
A=\left[\begin{array}{ccc}
\sum_{i=1}^{N_{1}}\left(F_{1, c}^{l}\right)^{T} F_{1, c}^{l}+\alpha_{1}^{l} & \ldots & \sum_{i=1}^{N_{1}} F_{1, c}^{l}\left(F_{i, c}^{l}\right)^{T} \\
\vdots & \vdots & \vdots \\
\sum_{i=1}^{N_{1}}\left(F_{i, c}^{l}\right)^{T} F_{1, c}^{l} & \cdots & \sum_{i=1}^{N_{1}}\left(F_{i, c}^{l}\right)^{T} F_{1, c}^{l}+\alpha_{1}^{l}
\end{array}\right]
$$

Finally, using the optimal solution of the conjugate gradient descent equation (5) and the convolution kernel can be updated according to the gradient descent method:

$$
\frac{\partial C_{2}(y)}{\partial f_{i, c}^{l}}=\sum_{i=1}^{N_{1}}\left(z_{i}^{l}\right)^{T}\left(\sum_{k=1}^{N_{2}} F_{k}^{\prime l} f_{i, c}^{l}-z_{i}^{l-1}\right)
$$




\subsection{Convolutional neural network image recognition training}

$\mathrm{CNN}$ includes convolution layer, pooling layer and full connection layer. Softmax classifier is used for multi task classification. According to the current mainstream CNN training techniques, nonlinear Relu activation function is used to enhance the sparsity and linear separability of features. The calculation of the characteristic images is as follows:

$$
z_{j^{\prime}}^{l}=f\left(\sum_{j \in M^{l}} z_{j}^{l-1} * f_{j j^{\prime}}^{l}\right)
$$

Where, $l$ is the number of layers of the convolution layer, $z_{\mathrm{j}}$ is the output characteristic graph $j$ of the layer $l, f_{\mathrm{j}}$ is the convolution kernel connecting the first characteristic graph $j$ of the layer $l-1$ and the second characteristic graph $j$ of the layer $l, M^{1}$ is the number of characteristic graphs of the layer $l-1, *$ is the convolution operator.

Because the distribution of input data changes after convolution operation, the standardized $\mathrm{BN}$ operation is introduced to process the data distribution. The $\mathrm{BN}$ is placed after the convolution operation of the network activation function, and the forward propagation convolution formula is transformed into:

$$
z_{j^{\prime}}^{l}=f\left(B N\left(\sum_{j \in M^{\prime}} z_{j}^{l-1} * f_{j j^{\prime}}^{l}\right)\right)
$$

The pooling layer downsamples the feature map of the previous convolution layer to obtain the output feature map with smaller dimension corresponding to the input feature map one by one :

$$
z_{j^{\prime}}^{l}=f\left(B N\left(\beta_{j^{\prime}}^{l} \operatorname{down}\left(z_{j^{\prime}}^{l-1}\right)\right)\right)
$$

Where: $\operatorname{down}()$ is the down-sampling function and $\beta$ is the down sampling coefficient.

The CNN model is trained by back propagation layer by layer, and the error cost function is:

$$
J(f)=\frac{1}{2} \sum_{i=1}^{N} \sum_{m=1}^{M}\left(\hat{y}_{m}^{i}-y_{m}^{i}\right)
$$

Finally, MB-SGD is used to update the weights:

$$
f_{j j^{\prime}}^{l}(t)=f_{j j^{\prime}}^{l}(t-1)-\frac{1}{N} \eta \sum_{i=1}^{N} \frac{\partial J}{\partial f_{j j^{\prime}}^{l}(t-1)}
$$

Where, $t$ represents the current time and $\eta$ represents the learning rate.

\subsection{Algorithm steps}

The input of the model in this paper is a training sample set of $N$ samples, and the output is the classification label and accuracy of the test sample in the fixed sample. The specific experiment process is as follows:

\subsubsection{Use deconvolutional network to extract feature mapping matrix}

First, use equation (1) to deconvolve the feature map $z_{\mathrm{i}}$ of the input image to obtain the reconstructed map $s_{\mathrm{i}}$. Then introduce auxiliary variable $\alpha_{\mathrm{i}}$, use formula (5) to obtain auxiliary variable $\alpha_{\mathrm{i}}$. Next, according to the obtained auxiliary variable $\alpha_{\mathrm{i}}$, use equation (6) to obtain the characteristic map, after multiple iterations of the above steps, use equation (7) to update the convolution kernel, and finally output the feature mapping matrix.

\subsubsection{Use $D M B D C N N$ for image fusion classification training}

At first, the feature map matrix extracted by the deconvolution network is weighted and fused as the initial matrix of the DMBDCNN convolution kernel, and the image data is convolved using formula (8). Secondly, use equation (9-10) to down-sampling and normalize the obtained convolution feature map. After training on each layer of the model, use formula (11-12) to supervise and fine-tune the convolutional network to update the model parameters.

\section{Experimental results and analysis}

Figure 3 shows the results of some original data after image enhancement. After data enhancement processing, the existing training samples will generate more training data.

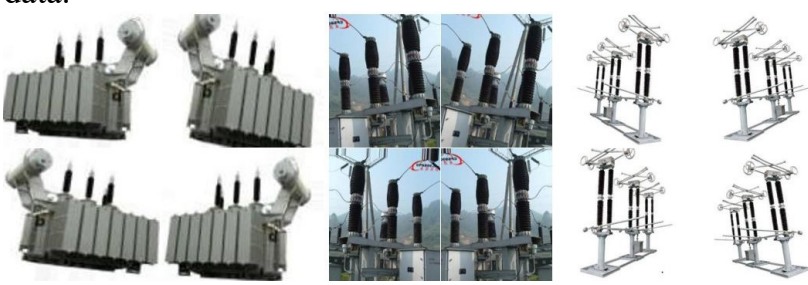

Figure 3. Some of the data enhances the presentation

This paper studies the classification and identification of transformer, circuit breaker and disconnector. A total of 1200 images are used for training and testing. The image size is set to $224 * 224 * 3$ after preprocessing, and each type of equipment has 400 images. During the training, the number of randomly selected images of transformer, circuit breaker and disconnector is 360 , and the remaining 40 are taken as test samples.

\subsection{Single-mode deconvolution network (SMDN) visible light image recognition}

For the classification of visible light electrical equipment images, this paper introduces the deconvolution feature extraction model. Compared with the traditional neural network classification algorithm, the model adjusts the number of convolution kernel layers to match the deconvolution feature extraction layer.

The freezing training method is adopted. Firstly, only the whole connection layer is trained. Secondly, unfreeze 
all layers for training. When using small batch to accelerate training, the batch size is set to 10 . The Dropout layer with the discard rate of 0.3 is introduced in the full connection layer 1 and 2, so as to avoid over fitting and freeze the learning rate of the first 100 freezing trainings is 0.001 , and the learning rate of the last 100 unfreezing trainings is 0.0001 . The classification accuracy of visible images is shown in Figure 4.

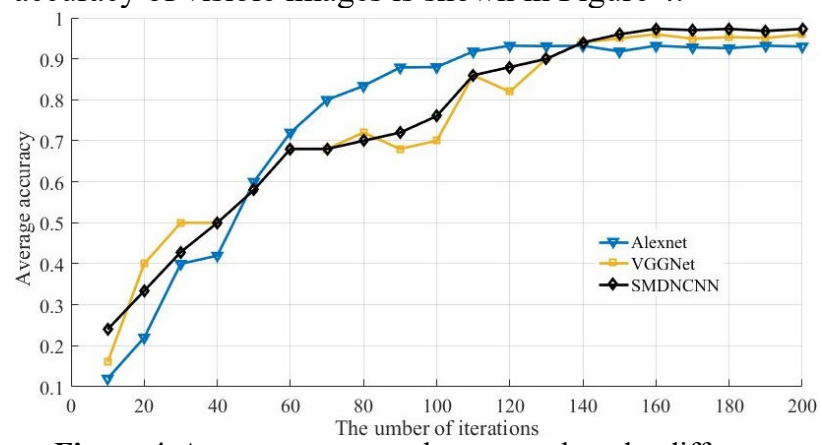

Figure 4. Average accuracy change graph under different training times

It can be seen from Figure 4 that compared with the traditional AlexNet and VGGNet, the average recognition accuracy of SMDNCNN has reached $97.32 \%$, indicating that the model in this paper can better adapt to the characteristics of fewer electrical equipment samples.

\subsection{Dual-mode visible and infrared image fusion recognition}

For electrical equipment visible and infrared image fusion classification, this paper introduces a weight coefficient $\mu$ after deconvolution feature extraction, uses the weighted fusion method for feature fusion. Different weight coefficients will affect the feature parameter matrix and affect the accuracy of image classification. Table 1 shows the influence of different weight coefficients $\mu$ on the classification accuracy rate.

Table 1. Classification accuracy $(\mu)$

\begin{tabular}{|c|c|c|c|c|c|c|}
\hline $\boldsymbol{\mu}$ & 0.1 & 0.5 & 0.9 & 1 & 3 & 5 \\
\hline Accuracy/\% & 97.8 & 98.8 & $\mathbf{9 9 . 1 7}$ & 88.1 & 33.3 & 33.3 \\
\hline
\end{tabular}

With the increase of the weight coefficient $\mu$, the classification accuracy gradually improves, and the weight coefficient $\mu=0.9$ is the critical value. Beyond this value, the accuracy decreases rapidly.

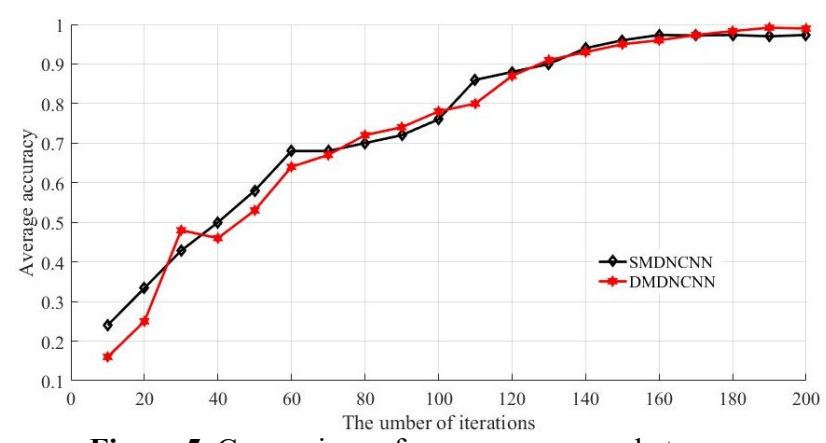

Figure 5. Comparison of average accuracy between SMDNCNN and DMDNCNN
It can be seen from Figure 5 that after 100 freezing training cycles and 100 thawing training cycles, compared with $97.3 \%$ of the single mode visible light image recognition accuracy, the accuracy of the dual mode fusion recognition reaches $99.17 \%$. The experimental results show that fusing the features extracted by deconvolution can highlight the unique information of different electrical equipment, and the parameter learning of neural networks can develop in a better direction. The further improvement of the average recognition accuracy rate illustrates this point.

\section{Conclusion}

Aiming at the local optimization and vanishing gradient problems that may be caused by the random initialization of the convolution kernel and the gradient descent learning method in the $\mathrm{CNN}$ training process, this paper proposes a convolutional network learning model based on deconvolutional feature extraction. The dual-mode deconvolution network (DMDN) used in this paper is able to automatically learn the feature mapping matrix of the image, which is used as the initial value of the convolution kernel to participate in the training of the $\mathrm{CNN}$ model. In addition, after weighted fusion of the learned visible light and infrared features, the stronger characteristic expression effectively solves the limitation of the limited number of samples. Compared to traditional model, the recognition accuracy of the improved model is reached at $99.17 \%$ after analysis and verification.

\section{Acknowledgments}

This paper was supported by the National Key R \& D Program of China (2017BJ0080).

\section{References}

1. Y.Q. Song, G.L. Zhou, Y.L. Zhu. Present Status and Challenges of Big Data Processing in Smart Grid. J. Power System Technology, 37(4): 927-935(2013)

2. J.F. Li, Q.R. Wang, M. Li. Electric Equipment Image Recognition Based on Deep Learning and Random Forest. J. High Voltage Engineering, 43(11): 3705-3711(2017)

3. H.R. Sun, Z.J. Pan, Y. Yan. Identification and location of photovoltaic hot spots with small samples based on deep convolutional autoencoder network. J. Journal of North China Electric Power University, 1-8(2021)

4. K. Xu, Z.J. Ling, Y.Y. Zhang. Image Recognition of Electric Equipment Based on GoogLeNet InceptionV3 Model. J. High Voltage Apparatus, 56(09): 129$135+143(2020)$

5. E.H. Lv, X.S. Wang, Y.H. Cheng. Deep convolution neural network learning based on deconvolution feature extraction. J. Control and Decision, 33(03): 447-454(2018) 